\title{
Towards demonstration of electron cooling with bunched electron beam
}

\author{
A. Fedotov
}

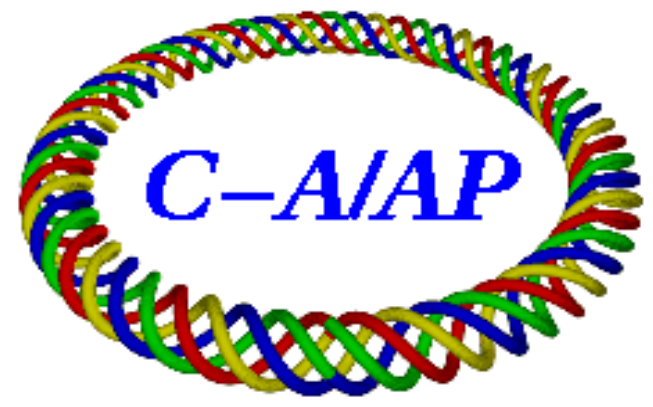

Collider-Accelerator Department Brookhaven National Laboratory Upton, NY 11973

Notice: This document has been authorized by employees of Brookhaven Science Associates, LLC under Contract No. DE-AC02-98CH10886 with the U.S. Department of En ergy. The United States Government retains a nonexclusive, paid-up, irrevocable, world-wide license to publish or reproduce the published form of this document, or allow others to do so, for United States Government purposes. 


\title{
TOWARDS DEMONSTRATION OF ELECTRON COOLING WITH BUNCHED ELECTRON BEAM
}

\author{
Alexei Fedotov \\ $C$-AD, Brookhaven National Laboratory
}

\section{Motivation}

All electron cooling systems which were in operation so far employed electron beam generated with an electrostatic electron gun in DC operating mode, immersed in a longitudinal magnetic field. At low energies magnetic field is also being used to transport electron beam through the cooling section from the gun to the collector. At higher energies (few MeV), it was shown that one can have simpler electron beam transport without continuous magnetic field. Because of a rather weak magnetic field on the cathode and in the cooling section the latter approach was referred to as "non-magnetized cooling", since there was no suppression of the transverse angular spread of the electron beam with the magnetic field in the cooling section. Such a cooler successfully operated at FNAL (2005-11) at electron beam energy of 4.3 MeV [1]. Providing cooling at even higher energies would be easier with RF acceleration of electron beam, and thus using bunched electron beam for cooling. Significant efforts were devoted to explore various aspects of such bunched electron beam cooling as part of R\&D of high-energy electron cooling for RHIC [2, 3]. However, experimental studies of such cooling are still lacking. Establishing this technique experimentally would be extremely useful for future high-energy applications.

Presently there is an ongoing effort to build Proof-of-Principle (PoP) experiment of Coherent Electron Cooling (CEC) at RHIC [4], which promises to be superior to conventional electron cooling for high energies [5]. Since the CEC experiment is based on bunched electron beam and it has sections where electron beam co-propagates with the ion beam at the same velocity, it also provides a unique opportunity to explore experimentally conventional electron cooling but for the first time with a bunched electron beam. As a result, it allows us to explore techniques needed for the high-energy electron cooling such as "painting" with a short electron beam and control of ion beam distribution under cooling which is essential if cooling is provided in a collider [6]. The software needed for comparison with the experiments is already developed as part of the previous high-energy electron cooling studies for RHIC [7]. Since electron beam will be non-magnetized and there will be no magnetic field in the cooling section it will be also a first demonstration of fully non-magnetized cooling.

The purpose of these studies was to explore whether we would be able to observe conventional electron cooling with parameters expected in the CEC PoP experiment. Below we summarize requirements on electron beam and cooling section needed for such demonstration.

\section{Parameters of electron beam and expected cooling performance}

Presently, the $112 \mathrm{MHz}$ SRF gun is being planned for the CEC experiment. The gun was already constructed by the Niowave company (Michigan) but it requires several modifications to be suitable for the CEC experiment, which are being planned. The repetition rate for the cooling demonstration will be only $78 \mathrm{kHz}$ to observe cooling on a single ion bunch in RHIC. For the CEC experiment electron beam will be accelerated to $21 \mathrm{MeV}$ energy needed for good amplification in the FEL (which employs undulator) and will provide cooling of gold ions at $40 \mathrm{GeV} / \mathrm{n}$. 
If one decides to use the CEC set-up in a parasitic mode without removing undulator and thus limited to only short sections of modulator or kicker for conventional electron cooling, the power of cooling is insufficient for studies at $\gamma=42.6$ at which the CEC experiment is being planned. A demonstration of high-energy electron cooling at such high energy with electron beam parameters expected in the CEC experiment would require using the CEC set-up in a dedicated mode (without undulator) which would allow to increase cooling section length to at least $13 \mathrm{~m}$.

To use the CEC set-up in a parasitic mode (without removing undulator) studies of bunch electron beam cooling can be performed at lower energies using the same set-up of the CEC experiment and electron bunches with relatively low peak current. The different options described above were studied in simulations using the BETACOOL code [7] and are summarized below.

First, we start with the baseline energy for the CEC experiment of $\gamma=42.6$. Even if one uses cooling section with effective cooling length of $13 \mathrm{~m}$ (dedicated mode), expected parameters of electron beam are only marginally sufficient to observe cooling. Parameters which were used in simulations are shown in Table 1 . In addition, in these simulations ion bunch intensity was reduced to $2 \mathrm{e} 8$ to minimize diffusion due to the Intra-beam Scattering (IBS). Demonstration of cooling at such high energy will require strict control on electron angular spread in the cooling section. With the largest contribution to the angular spread coming from beam emittance, it puts challenging requirement on achieving rather small beam emittance from the gun. Producing electron beam with smaller emittance will enhance cooling performance as shown in Figs. 1-3 for $1 \mathrm{nC}$ bunch charge with the normalized rms emittance of electron bunch of 3,2 and $1.3 \mu \mathrm{m}$, respectively.

If very small emittance of $1.3 \mu \mathrm{m}$ is achieved then cooling demonstration may be possible even with smaller charge of $0.75 \mathrm{nC}$, as shown in Fig. 4. Note that cooling performance presented in Figs. 1-4 should be seen as for "demonstration purpose only". Actual cooling will strongly depend on electron beam characteristics obtained from the $112 \mathrm{MHz}$ SRF gun (which are presently unknown) with an accurate estimate and optimization of various contributions to the angular spread of electron beam, as well as electron beam optics, in the cooling section. One can see that despite electron bunch length being very short (only $1 \mathrm{~mm} \mathrm{rms}$ ) ions at significantly larger amplitudes are also being effectively cooled as a result of the synchrotron and betatron oscillations (in Figs. 1-4, the rms length of ion bunch is $75 \mathrm{~cm}$ ).

\begin{tabular}{|l|l|}
\hline Electron kinetic energy, $\mathrm{MeV}$ & 21 \\
\hline Length of cooling section, $\mathrm{m}$ & 13 \\
\hline Charge per bunch, $\mathrm{nC}$ & $0.75-1.0$ \\
\hline Electron peak current, $\mathrm{A}$ & $90-119$ \\
\hline Rms length of electron bunch, $\mathrm{mm}$ & 1 \\
\hline Rms emittance, $\mu \mathrm{m}$ & $\leq 3$ \\
\hline Rms momentum spread & 0.001 \\
\hline
\end{tabular}

Table 1. Parameters of electron beam for demonstration of conventional electron cooling at $\gamma=42.6$ based on the CEC experimental set-up. 


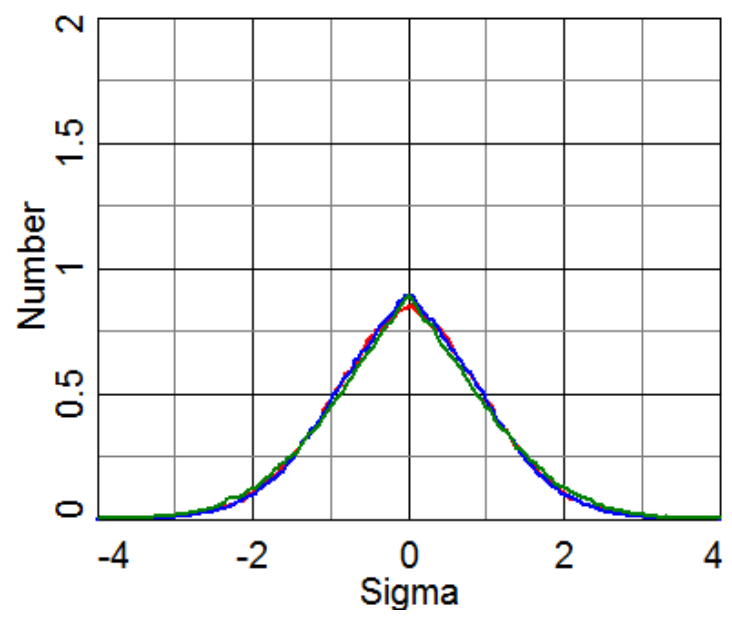

Fig. 1. Longitudinal (green; rms length $\sigma=75 \mathrm{~cm}$ ) and transverse (blue and red) ion bunch profiles after $900 \mathrm{~s}$ of cooling at $40 \mathrm{GeV} / \mathrm{n}$ with electron bunch charge of $1.0 \mathrm{nC}$ (peak current of $119 \mathrm{~A}$ ), rms normalized emittance of $3 \mu \mathrm{m}$ and parameters given in Table 1 .

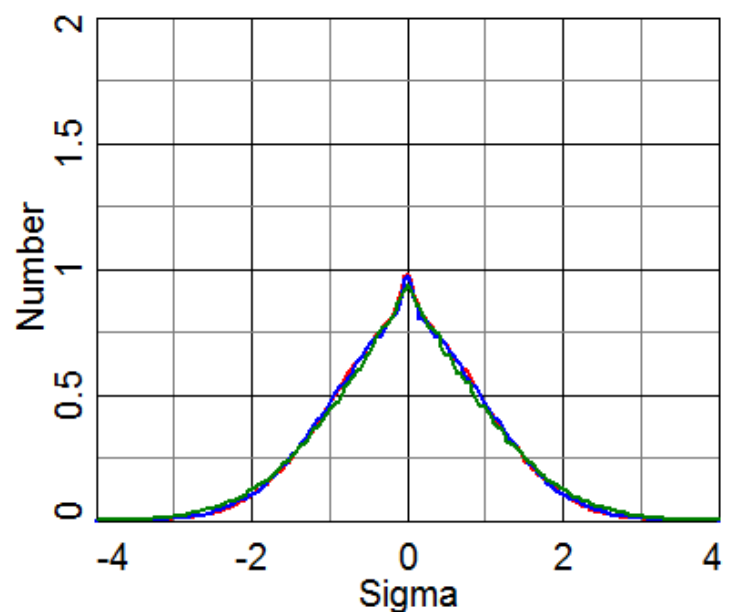

Fig. 2. Longitudinal (green; rms length $\sigma=75 \mathrm{~cm}$ ) and transverse (blue and red) ion bunch profiles after $900 \mathrm{~s}$ of cooling at $40 \mathrm{GeV} / \mathrm{n}$ with electron bunch charge of $1.0 \mathrm{nC}$ (peak current of $119 \mathrm{~A}$ ), rms normalized emittance of $2 \mu \mathrm{m}$ and parameters given in Table 1 .

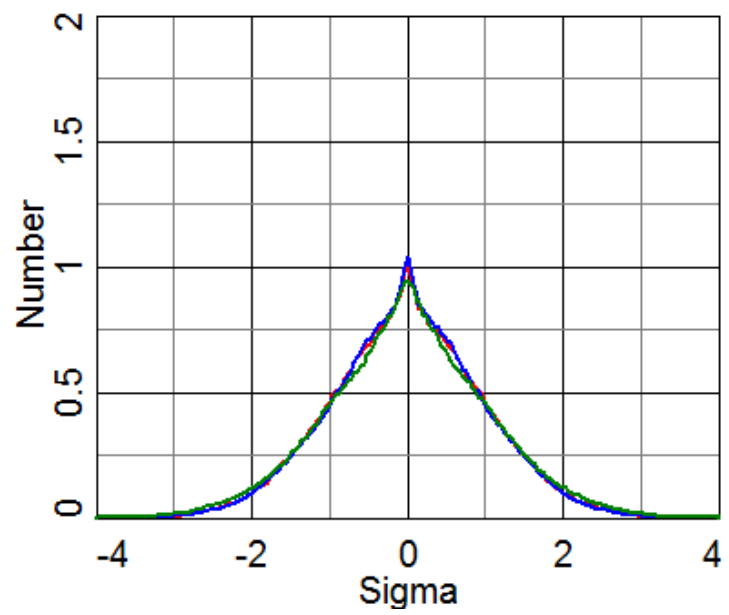

Fig. 3. Longitudinal (green; rms length $\sigma=75 \mathrm{~cm}$ ) and transverse (blue and red) ion bunch profiles after $900 \mathrm{~s}$ of cooling at $40 \mathrm{GeV} / \mathrm{n}$ with electron bunch charge of $1.0 \mathrm{nC}$ (peak current of $119 \mathrm{~A}$ ), rms normalized emittance of $1.3 \mu \mathrm{m}$ and parameters given in Table 1. 


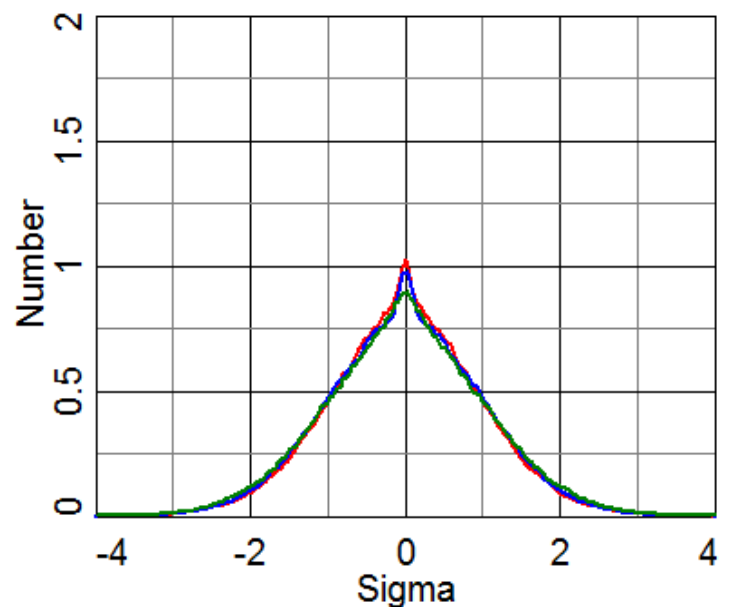

Fig. 4. Longitudinal (green) and transverse (blue and red) ion bunch profiles after $900 \mathrm{~s}$ of cooling at $40 \mathrm{GeV} / \mathrm{n}$ gold ions beam energy with electron bunch charge of $0.75 \mathrm{nC}$ (peak current of $90 \mathrm{~A}$ ), rms normalized emittance of $1.3 \mu \mathrm{m}$ and parameters given in Table 1.

Since electron cooling power strongly increases with decrease of beam energy, demonstration of such cooling would be easier at lower energies. At lower energies such demonstration would be possible even in a parasitic mode for the CEC experiment using only short cooling section of a modulator, for example.

The typical injection energy of gold ions in RHIC is at $\gamma=10.6$ which makes it convenient for this experiment with ions at injection energy being readily available during the RHIC operation. At such low energy, there is no requirement to have very high peak current in electron bunch. For experiment at $\gamma=10.6$ the peak current of about 10 A could be sufficient. In fact, at such low energy even 10 A current can already produce significant contribution to the transverse angular spread in the cooling section due to the space charge, and compensating solenoids may be needed. As a result, long electron bunches are preferred. Acceleration and beam transport of such long bunches should be compatible with the present design for the CEC experiment.

\begin{tabular}{|l|l|}
\hline Electron kinetic energy, $\mathrm{MeV}$ & 4.9 \\
\hline Length of cooling section, $\mathrm{m}$ & 3 \\
\hline Charge per bunch, $\mathrm{nC}$ & $0.75-1.5$ \\
\hline Electron peak current, $\mathrm{A}$ & $\geq 10$ \\
\hline Full length of electron bunch, $\mathrm{ps}$ & $>100$ \\
\hline Rms emittance, $\mu \mathrm{m}$ & 3 \\
\hline Rms momentum spread & 0.001 \\
\hline
\end{tabular}

Table 2. Parameters of electron beam for demonstration of bunched electron beam cooling at $\gamma=10.6$ based on the CEC experimental set-up.

Figure 5 shows that it will be difficult to study conventional cooling with electron bunch charge of only $0.75 \mathrm{nC}$ and 9 A peak current. After $900 \mathrm{sec}$ of cooling only very small change in the longitudinal distribution is observed. However, cooling is more effective for the same peak current but twice longer bunch length, as shown in Fig. 6. Increasing peak current above $10 \mathrm{~A}$ would help cooling even further but this would require stronger control of electron angular spread due to the space charge. For example, for bunch charge of $1.5 \mathrm{nC}$ and peak current of $18 \mathrm{~A}$ resulting 
longitudinal and transverse ion beam distributions are shown in Fig. 7. However such electron beam peak current of $18 \mathrm{~A}$ will require short compensating solenoids every $1 \mathrm{~m}$ of beam transport.

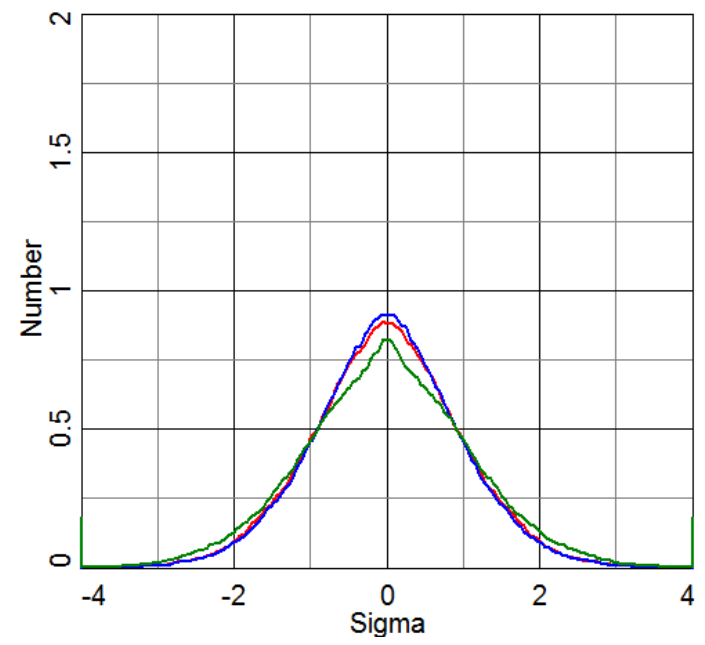

Fig. 5. Longitudinal (green) and transverse (blue and red) ion bunch profiles at $10 \mathrm{GeV} / \mathrm{n}$ with electron bunch charge of $0.75 \mathrm{nC}$, peak current of $9 \mathrm{~A}$ and parameters given in Table 2 .

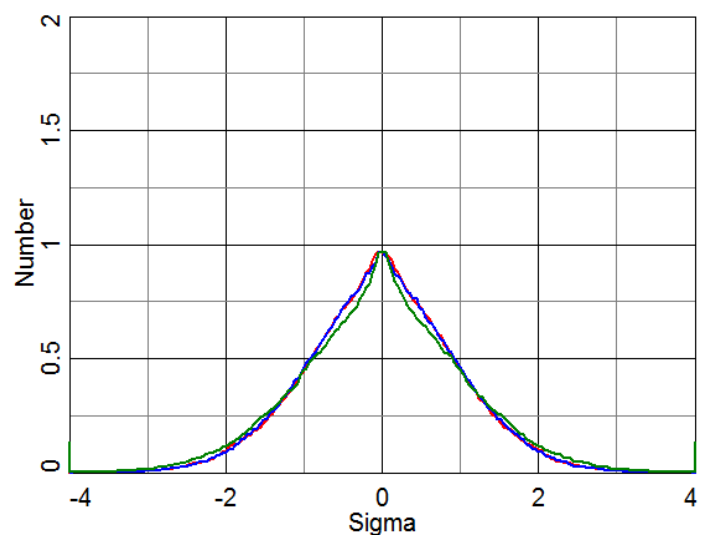

Fig. 6. Cooling of the longitudinal (green) and transverse (blue and red) ion bunch profiles at 10 $\mathrm{GeV} / \mathrm{n}$ with 9 A peak current but longer bunch length (assuming larger charge per bunch) and parameters given in Table 2 .

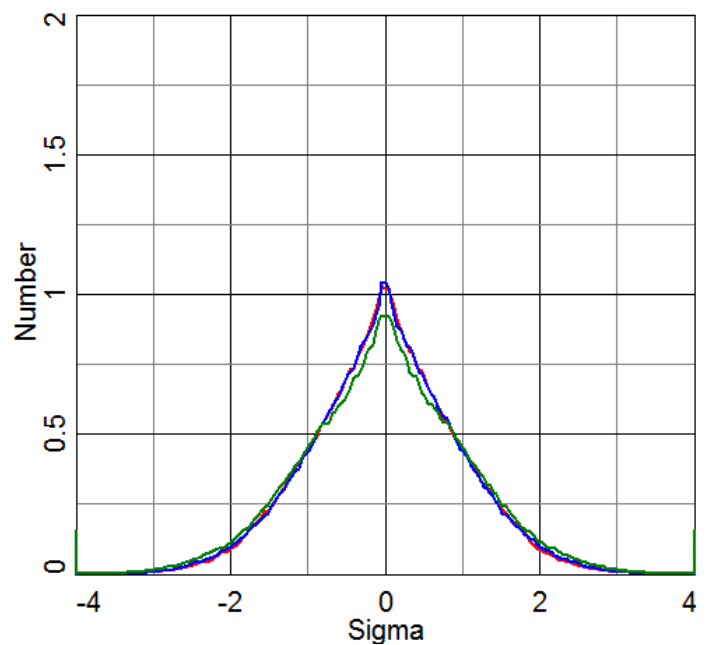

Fig. 7. Cooling of the longitudinal (green) and transverse (blue and red) ion bunch profiles at 10 $\mathrm{GeV} / \mathrm{n}$ with electron beam charge of $1.5 \mathrm{nC}$ and parameters given in Table 2 . 
If necessary, experiment can be performed at even lower energies. Table 3 shows example for $\gamma=4.1$ for which RHIC operation set-up is available (c.m. energy $7.7 \mathrm{GeV} / \mathrm{n}$ ). An advantage of this energy is that required electron energy of $1.6 \mathrm{MeV}$ should be available directly from the gun and no additional acceleration is needed. However, beam angular spread due to the space charge becomes even stronger. As a result, bunch length should be longer than 100 ps or short compensating solenoids should be used in the beam transport and cooling section. The questions which should be addressed, if such low energy is selected for the demonstration experiment, is production of long electron bunches (for example $500 \mathrm{ps)} \mathrm{and} \mathrm{design} \mathrm{of} \mathrm{electron} \mathrm{beam} \mathrm{transport} \mathrm{at} \mathrm{this} \mathrm{energy} \mathrm{which}$ does not lead to a significant degradation of beam emittances and energy spread.

\begin{tabular}{|l|l|}
\hline Electron kinetic energy, $\mathrm{MeV}$ & 1.6 \\
\hline Length of cooling section, $\mathrm{m}$ & 3 \\
\hline Charge per bunch, $\mathrm{nC}$ & $<1.0$ \\
\hline Electron peak current, $\mathrm{A}$ & $<7.5 \mathrm{~A}$ \\
\hline Full length of electron bunch, ps & $>100$ \\
\hline Rms emittance, $\mu \mathrm{m}$ & 3 \\
\hline Rms momentum spread & 0.001 \\
\hline
\end{tabular}

Table 3. Parameters of electron beam for demonstration of conventional electron cooling at $\gamma=4.1$ based on the CEC experimental set-up.

\section{Summary}

The CEC PoP experiment which is presently under design at Collider-Accelerator department at BNL may also be suitable for the demonstration of conventional electron cooling but for the first time with a bunched electron beam, which is essential for future high-energy applications. To provide such a possibility, one needs to make sure that present design and electron beam parameters of the CEC experiment are compatible with the parameters needed for conventional electron cooler. Present studies showed that several scenarios for demonstration of conventional electron cooling with bunched electron beam may be possible.

For cooling of gold ions at $40 \mathrm{GeV} / \mathrm{n}$ energy, which is the baseline energy for the CEC experiment, one would need to use full available free space as a cooling section (13 $\mathrm{m}$ was assumed in these studies). In addition, it will be essential to achieve rather small emittance of electron beam for expected bunch charge of around $1 \mathrm{nC}$. On the other hand, a rather short cooling section and somewhat relaxed requirements on electron beam emittance are needed for the demonstration of bunched electron beam cooling at lower energies.

In addition to being first demonstration of bunched beam electron cooling, such electron cooling experiment would allow us to explore techniques needed for the high-energy electron cooling such as "painting" with a short electron beam and control of ion beam distribution under cooling which is essential if cooling is provided in a collider. Since electron beam will be fully nonmagnetized and there will be no magnetic field in the cooling section it will be also first demonstration of the fully non-magnetized cooling.

If cooling is performed at $40 \mathrm{GeV} / \mathrm{n}$, this would be also demonstration of the highest energy electron cooling, by far. In addition it would establish feasibility of using conventional bunched beam electron cooling above transition energy in RHIC which could be used for pre-cooling of both heavy ions and protons for future RHIC operation or for eRHIC (actual pre-cooling of all ion bunches for RHIC operation would require upgrade of electron beam injector), if needed [8, 9]. 


\section{Acknowledgement}

We would like to thank D. Kayran and V. Litvinenko for useful discussions on this subject.

\section{References}

[1] S. Nagaitsev et al., PRL 96, 044801 (2006).

[2] I. Ben-Zvi, "Status of the R\&D towards electron cooling of RHIC", Proc. of PAC07 (Albuquerque, New Mexico), p. 1938.

[3] A. Fedotov, "Progress of high-energy electron cooling for RHIC", Proc. of COOL07 (Bad Kreuznach, Germany), MOM2103, p. 11.

[4] V.N. Litvinenko et al., Proc. of PAC'11 (New York), p. 2064, THOBN3.

[5] V.N. Litvinenko and Ya. Derbenev, PRL 102, 114801 (2009).

[6] A. Fedotov, I. Ben-Zvi, D. Bruhwiler, V. Litvinenko, A. Sidorin, "High-energy electron cooling in a collider", New Journal of Physics 8 (2006), p. 283.

[7] BETACOOL code, http://lepta.jinr.ru; A. Sidorin et al., NIM A 558, p. 325 (2006).

[8] A. Fedotov, BNL C-AD Tech Note C-A/AP/333, October 2008.

[9] A. Fedotov, "Pre-cooling for eRHIC" (eRHIC meeting, January 2008). 